\title{
Crystallization kinetics of cephalexin monohydrate in the presence of cephalexin precursors
}

Matthew A. McDonald, Grant D. Marshall, Andreas S. Bommarius, Martha A. Grover,

Ronald W. Rousseau*

School of Chemical and Biomolecular Engineering, Georgia Institute of Technology,

Atlanta GA, 30332

\section{Supplemental Material}

\section{Contents}

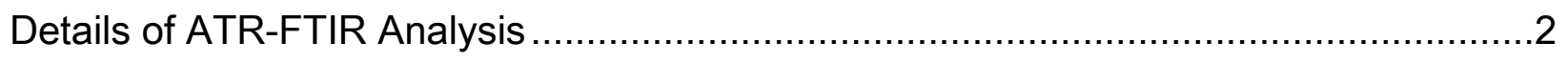

Details of Simulated Crystallization Experiment ...................................................

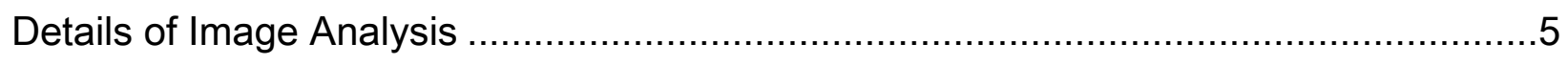

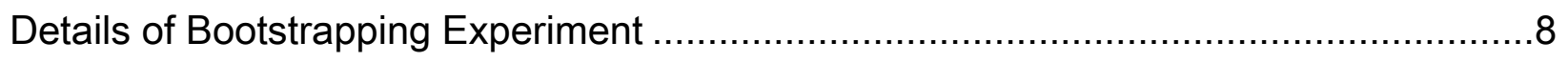

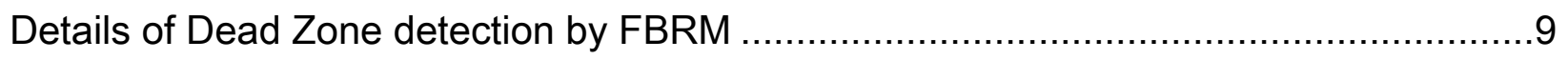

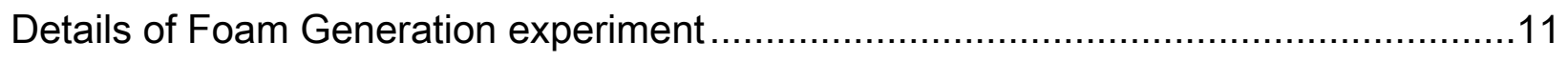

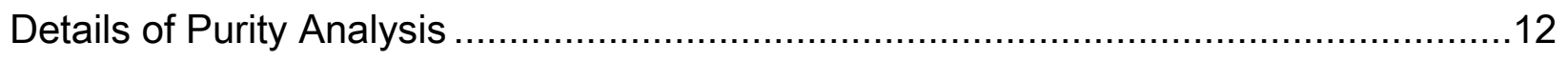


Comparison of primary and secondary nucleation 


\section{Details of ATR-FTIR Analysis}

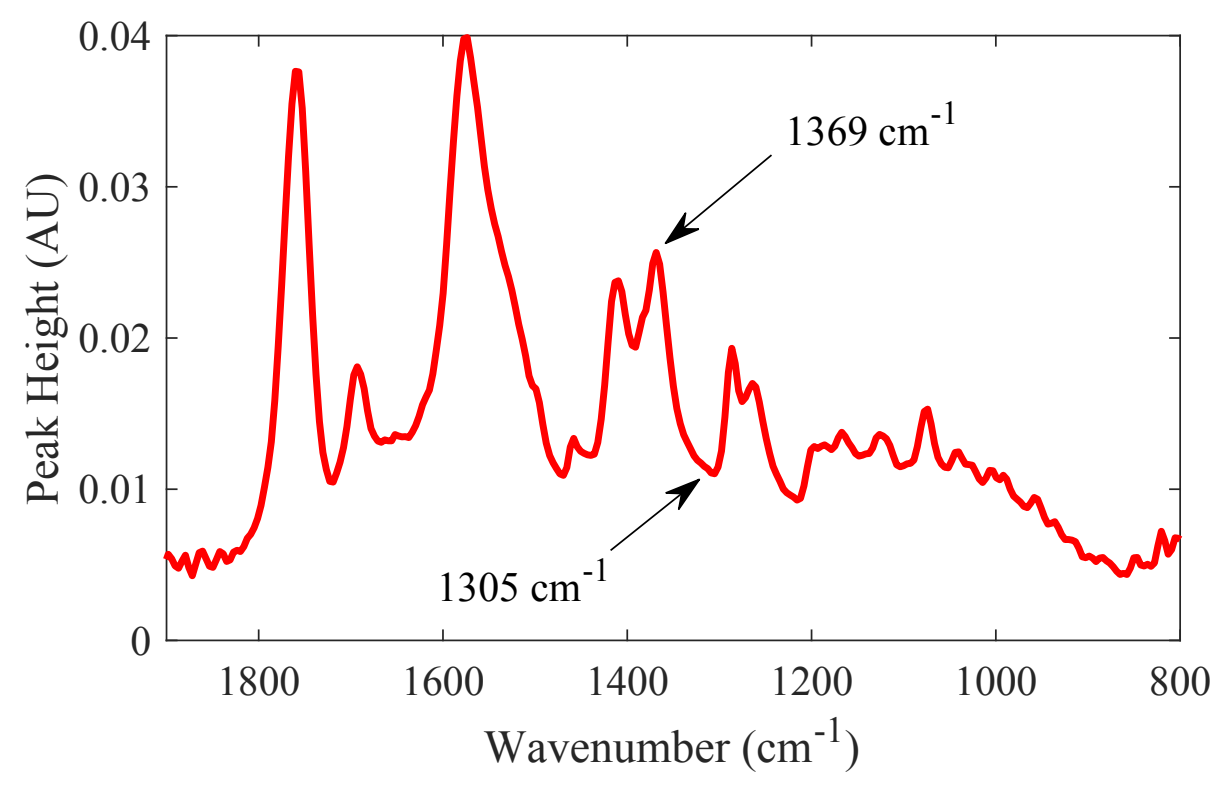

Figure S1. The IR spectrum of cephalexin in water is shown after subtracting the water baseline. The peak and trough at 1369 and 1305 were used to quantify the concentration of the cephalexin in solution. The larger peaks gave less reproducible concentration measurements.

\section{Details of Simulated Crystallization Experiment}

A suspension of crystals produced by the method described in the main text was incrementally added to a saturated solution of cephalexin and the resulting increase in FBRM counts was observed. The counts and suspension density do not linearly correlate, 
the counts saturate as the suspension density increases. This observation has previously been termed the snowstorm effect where a flurry of small particles obscures the FBRM from sampling the true crystal size distribution. The rapid onset of the snowstorm effect at low weight fraction of solids was likely due to the needle-like morphology and small average size of the cephalexin crystals. A linear approximation of the count-suspension density relationship could be made below approximately 40,000 counts, where each count per second corresponded to approximately $1.5 \pm 0.2$ million crystals per liter. The number density of crystals in suspension was estimated by image analysis with a hemocytometer (see supplemental Figure S3).

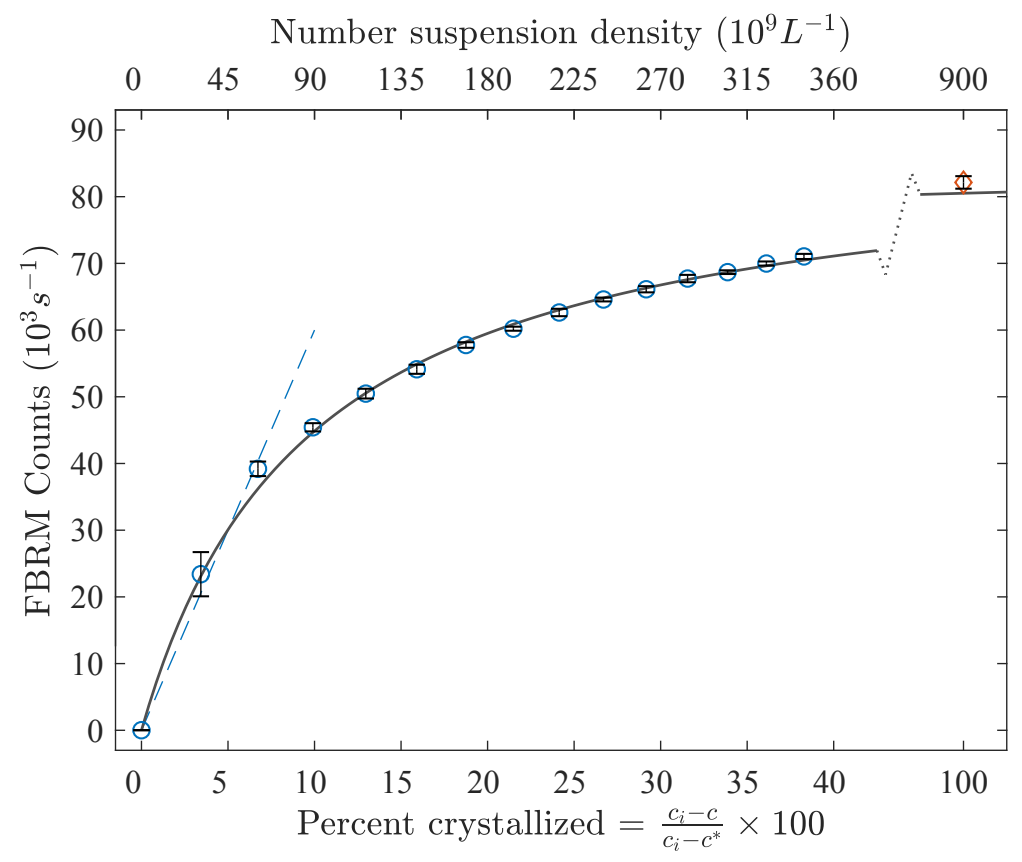


Figure S2. The number of crystals in suspension, as measured by extent of crystallization (bottom axis) and offline microscopy (top axis), was correlated with the number of chord counts observed online by FBRM. The FBRM counts were fit with a hyperbolic trend (solid curve) and initial linear trend (dashed line). Note the break in the axis for the final measured point.

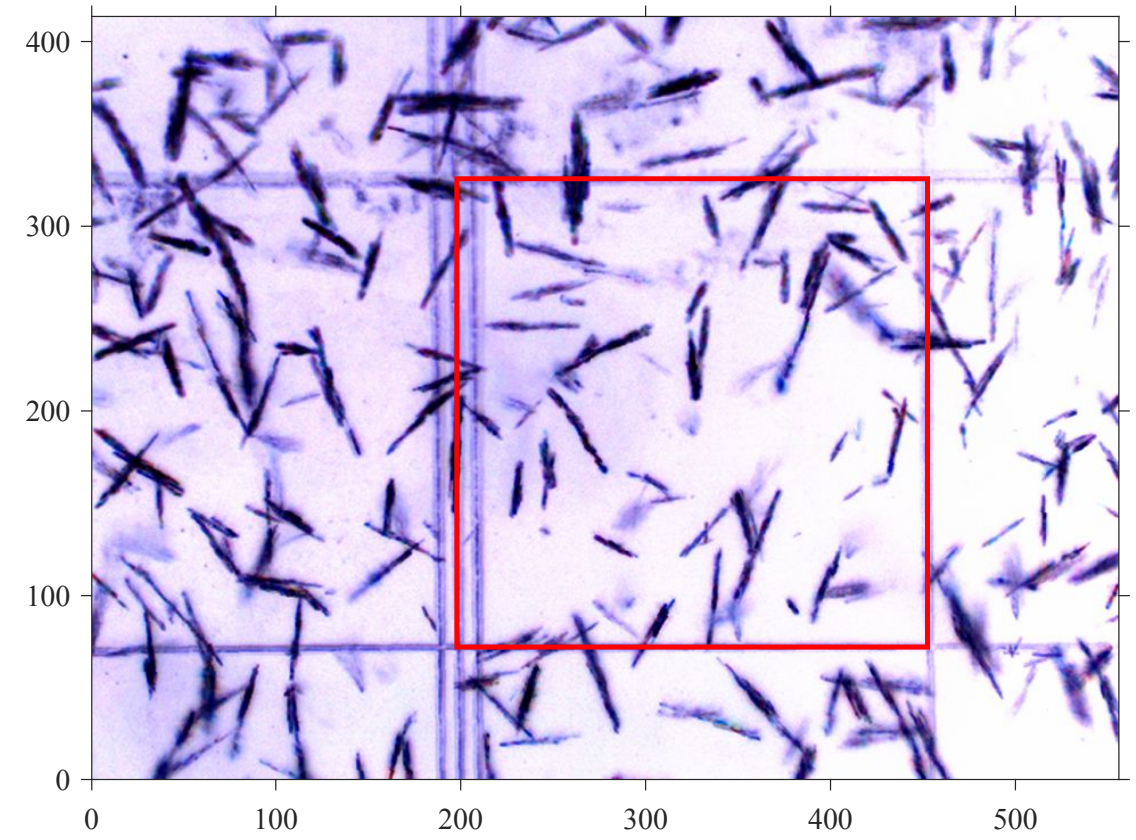

Figure S3. A hemocytometer counting chamber was used to determine the crystal number suspension density with offline image analysis. A 1/100 dilution (in saturated solution) is shown. The area inside the red square has a volume of $6.25 \mathrm{~nL}$ and 
contains 51 crystals (counting those on the left and top borders but discounting those on the right and bottom borders). Using several images and randomly sampling different squares in the same image the total number density was determined to be $890 \pm 89$ billion crystals per liter for this sample. This image was sampled from the end of a batch of cephalexin with no co-solute and an initial supersaturation of 2.56. 
Details of Image Analysis
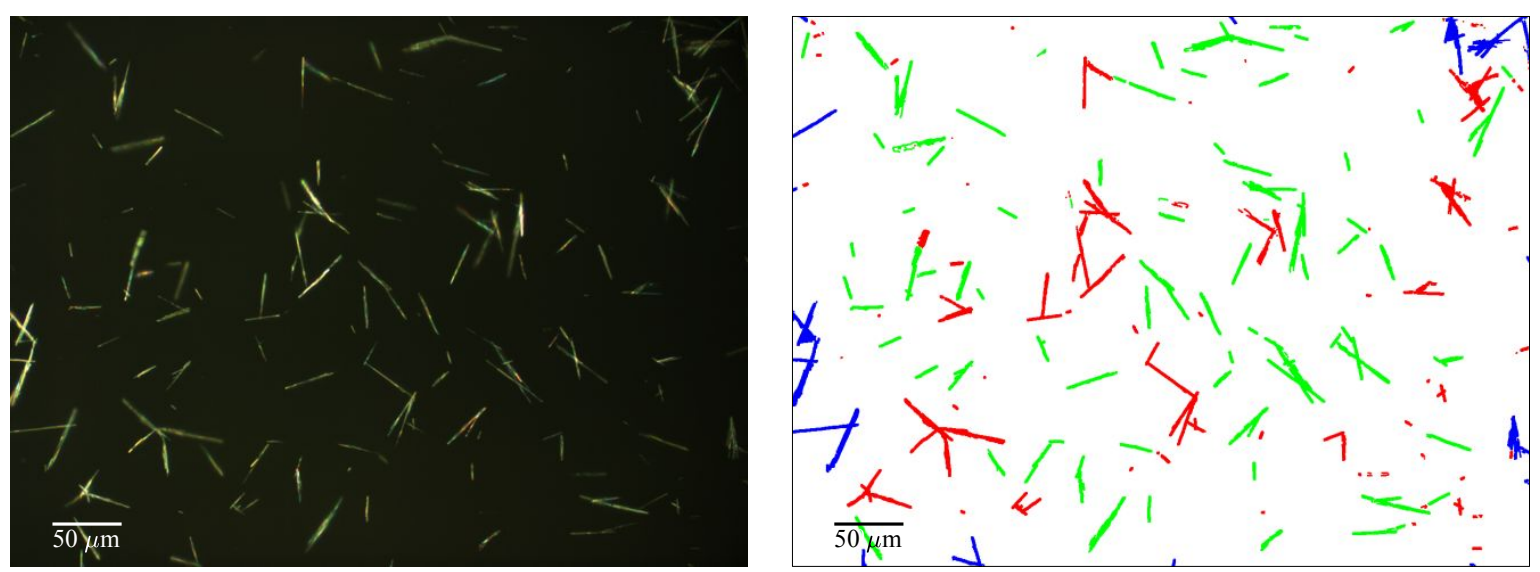

Figure S4. An optical micrograph of product cephalexin crystals (left) and the result of processing that image (right), in addition to Figure 2 in the main text.

A sample of the product slurry was withdrawn at the end of each experiment and diluted in saturated cephalexin solution to allow individual crystals to be distinguished in the microscope images; the amount of dilution was critical to generating an accurate CSD. In Figure S4 the suspension was not diluted enough; $53 \%$ of the crystals were excluded from the CSD because they overlap or touch the border of the frame. In figure 2 in the main text only $40 \%$ of the crystals were excluded from the CSD. 
MATLAB was used to process the images as follows. First a threshold filter was applied to flatten the background and avoid detecting artifacts and out of focus object. Then edges were detected using the Sobel operator edge detection algorithm. Gaps in the edges were filled by dilating the edge image with linear structuring elements such that edges aligned end-to-end would connect (e.g. a perceived gap in a crystal where the crystal is really one continuous entity) but those that met at angles or at a midpoint in the crystal would not connect. Holes in the interior of crystals were filled and the length and width of each crystal was measured. Crystals that touched the border of the image (blue) were not counted because their true size is unknown. Crystals were filtered by their aspect ratio, and crystals with an aspect ratio below 3.5 (red) were not counted because they usually represented overlapping or clumped crystals. The crystals used to construct a CSD (green) were not always single crystals if a large and small crystal overlapped. The bias created by uncounted small crystals (because they overlap a counted large crystal) was offset by the bias resulting from the higher chance of large crystals overlapping. 


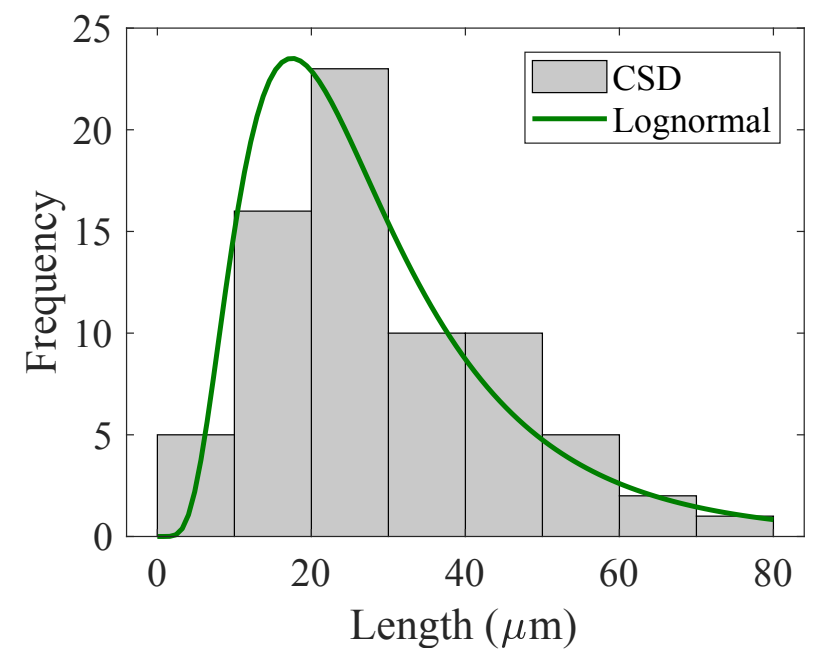

Figure S5. The CSD calculated from Figure S4. The CSD histogram was fit to a lognormal distribution (green curve).

The lognormal probability distribution function, $f(x)$, is defined in Equation $\mathrm{S} 1$, with parameters $\bar{m}$ and $\sigma$.

$$
f(x)=\frac{1}{x \sigma \sqrt{2 \pi}} \exp \left[\frac{-(\ln x-\bar{m})^{2}}{2 \sigma^{2}}\right]
$$

The CSD measured by image analysis was fit to the lognormal distribution with the MATLAB function lognfit. The moments of the CSD, as defined in Equations 4 and 5 , are related to the lognormal parameters by Equation $\mathrm{S} 2$. 


$$
\ln \left(\frac{\mu_{i}}{\mu_{0}}\right)=\bar{m} i+\frac{1}{2} \sigma^{2} i^{2} \quad \forall i=0,1, \ldots, N_{\mu}
$$




\section{Details of Bootstrapping Experiment}

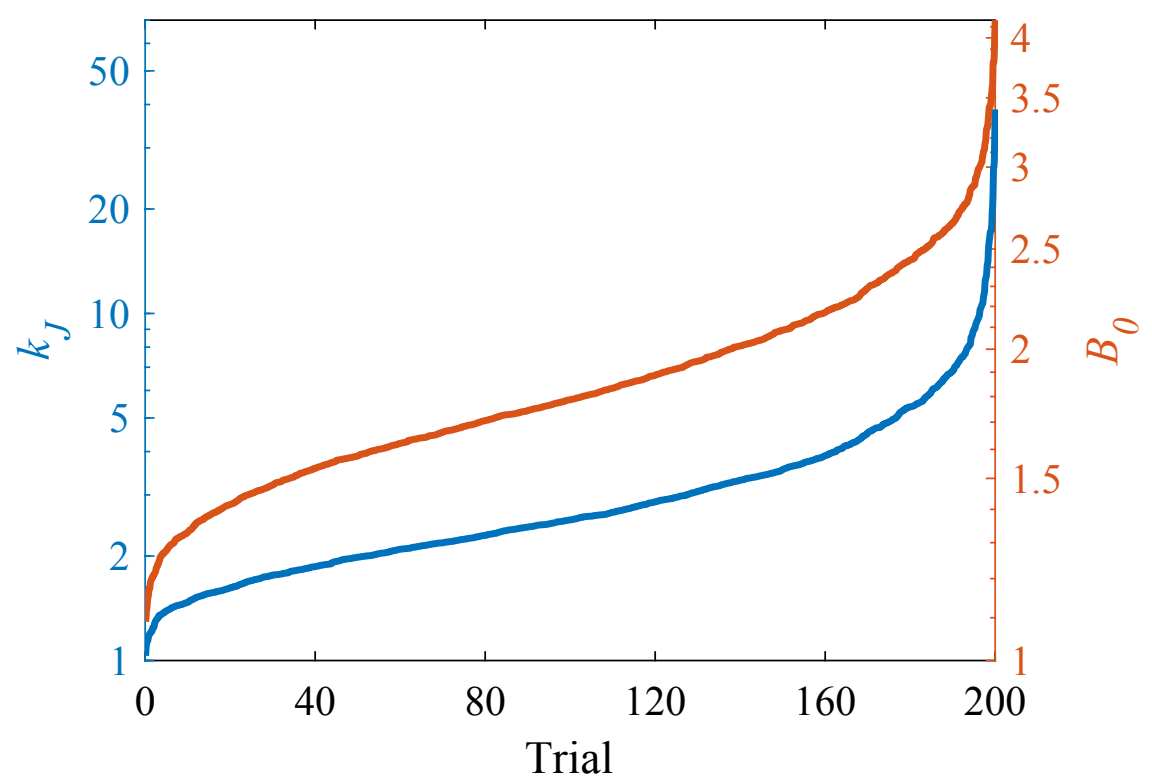

Figure S6. Bootstrapping was used to determine the primary nucleation parameters. In this figure the parameters that gave the best fit to Equation 2 (for data randomly generated by taking four samples from each of three data sets at different supersaturation values) are sorted and plotted for 200 bootstrap trials. The confidence interval lies between the $2.5^{\text {th }}$ and $97.5^{\text {th }}$ percentile. 


\section{Details of Dead Zone detection by FBRM}
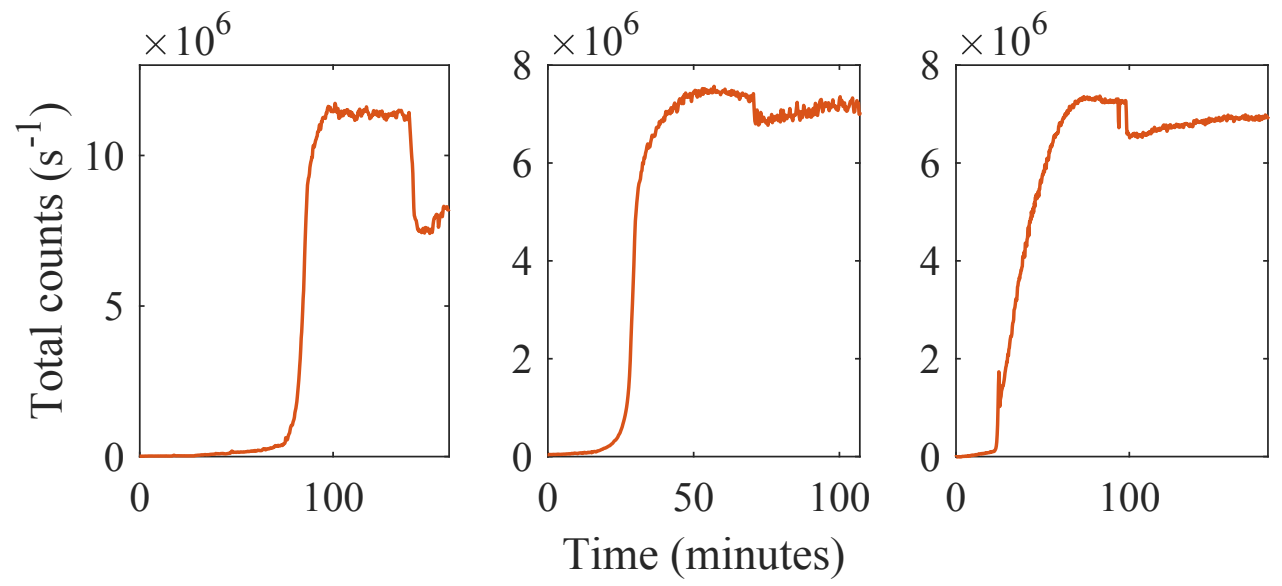

Figure S7. Three examples of anomalous FRBR readings suggesting that a dead zone had formed in front of the FBRM probe window at (from left to right) 140 minutes, 70 minutes, and 100 minutes. In all experiments, the counts followed an s-shape curve in time, eventually saturating when the snowstorm effect prevents the FBRM from detecting all crystals. In the experiments where a dead zone formed, the counts rapidly drop, suggesting crystals had stopped moving in front of the probe window.

A tracer experiment was used to visualize dead zones. Dye was added to an experiment where the FBRM had indicated a dead zone may have formed (see Figure S7). Through the observation window in the Mettler-Toledo OptiMax it could be seen 
that the dye had only entered into the well-mixed region of the reactor, which was colored, while the poorly mixed region remained milky white (due to the dense cephalexin monohydrate slurry). This confirmed that the anomalous FBRM reading was the result of dead zone formation, and that all experiments that showed an anomalous FBRM reading should not be considered for the determination of crystallization kinetics.

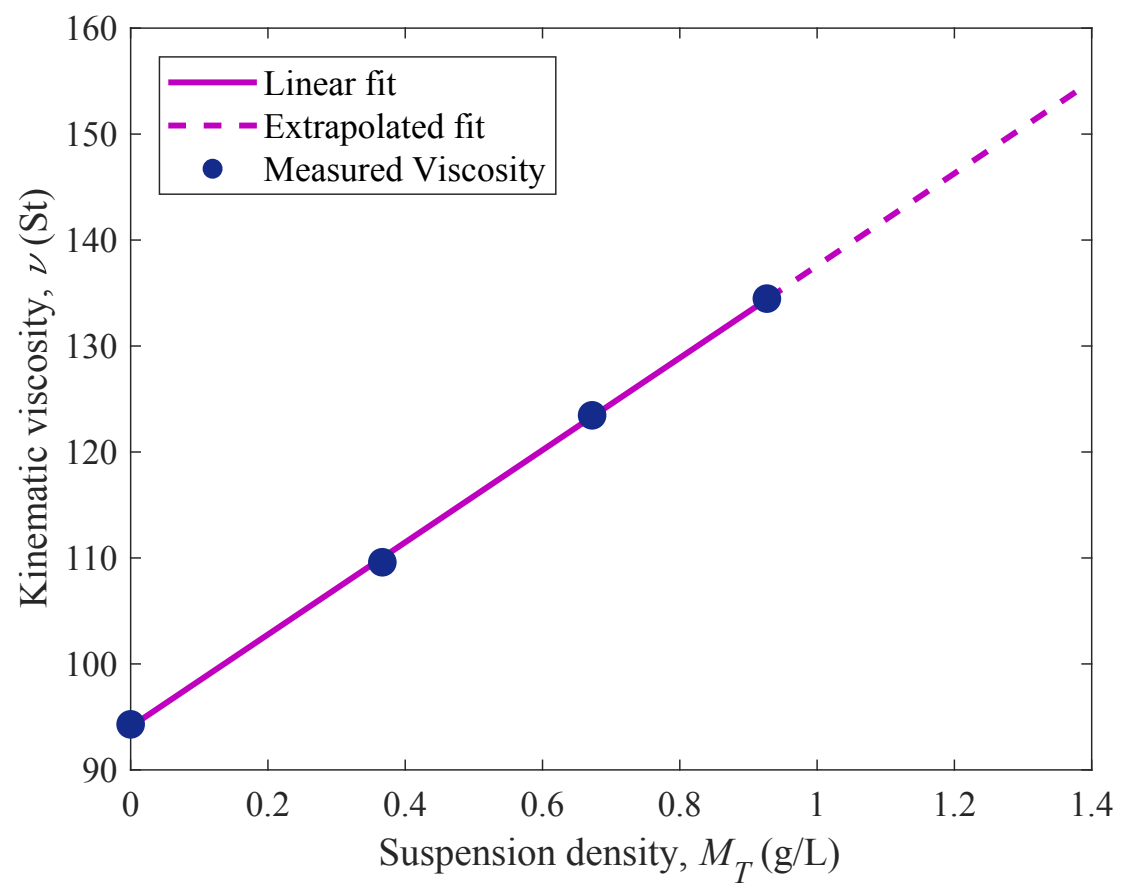

Figure S8. The kinematic viscosity as a function of suspension density was measured with a capillary viscometer at different suspension densities, including a filtered solution of mother liquor. The viscometer did not generate accurate measurements above 1.0 
$\% \mathrm{w} / \mathrm{v}$ because the crystals blocked the capillary. The viscosity was found to vary

linearly with suspension density, and this linear correlation was extrapolated to suspension densities above the limit of measurement. 
Details of Foam Generation experiment

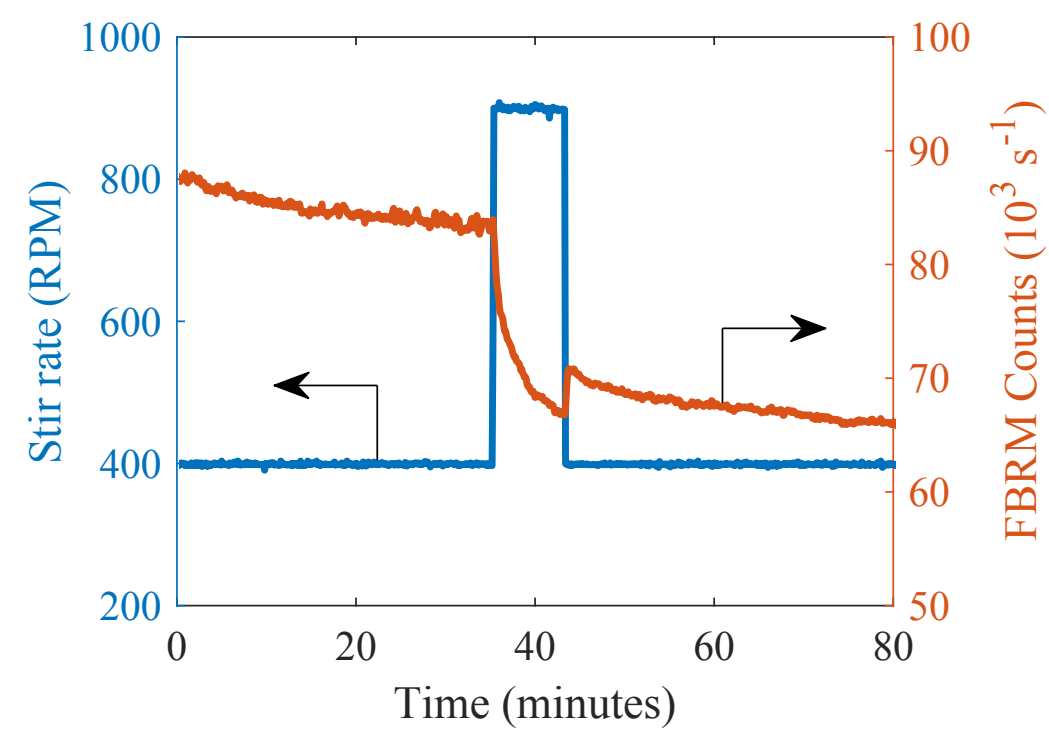

Figure S9. The stir rate (blue) was increased from 400 to 900 RPM for 5 minutes to generate a stable foam. The counts measured by FBRM (red) decreased during foam generation and did not return after the stir rate was dropped back to 400 RPM, indicating crystals had been permanently sequestered in the foam. 
Details of Purity Analysis

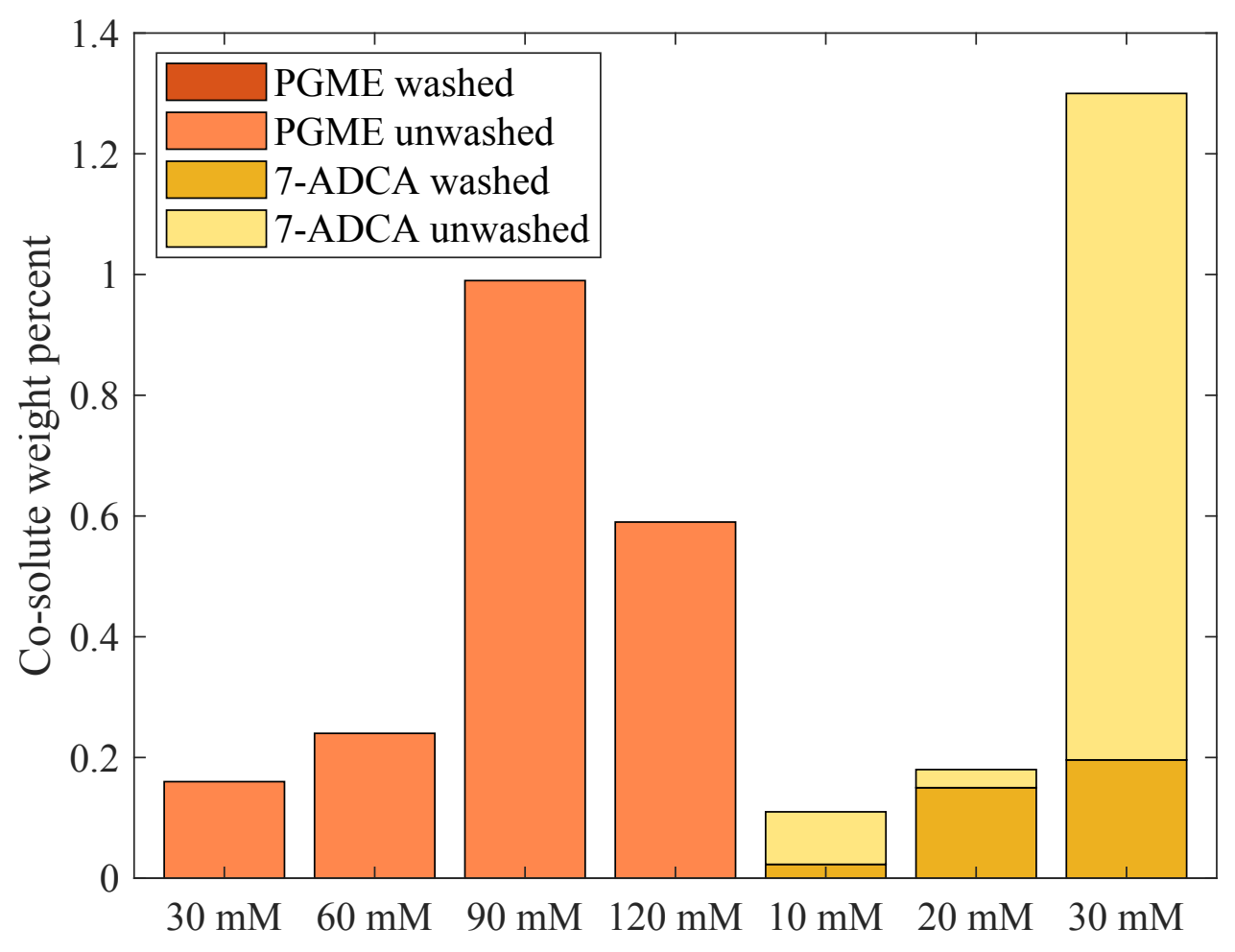

Figure S10. HPLC analysis of purity of unwashed (light hue) and washed (dark hue) cephalexin monohydrate crystals formed in the presence of a co-solute at different concentrations. The $50 \mathrm{mM}$ 7-ADCa point was excluded because 7-ADCA also crystallized giving a physical mixture of solid 7-ADCA and cephalexin monohydrate (consisting of roughly $30 \%$ 7-ADCA). Washing consisted of suspending $250 \mathrm{mg}$ of 
product cephalexin in $5 \mathrm{~mL}$ of cold water $\left(<5^{\circ} \mathrm{C}\right)$ and stirring for 15 minutes under chilled conditions. 
Comparison of primary and secondary nucleation

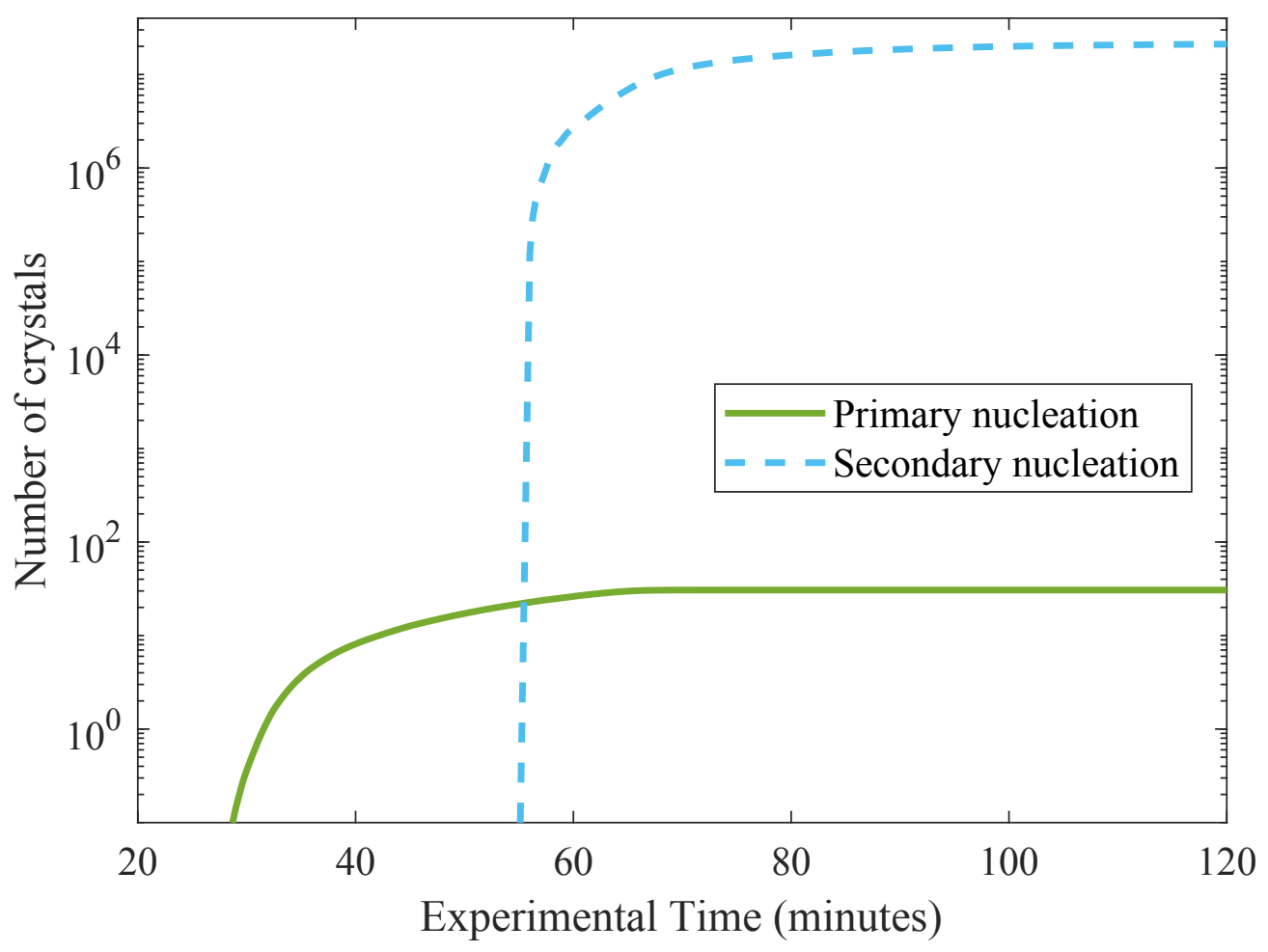

Figure S11. Comparison of crystals born from primary nucleation (green solid curve)

and secondary nucleation (blue dashed curve). The curves were generated with

Equation 2 (primary) and 7 (secondary) the parameters in Table 2 and the

supersaturation profile in Figure 5. Note the logarithmic scale. 